\title{
Mapping the spatial distribution and changes of oil palm land cover using an open source cloud-based mapping platform
}

\begin{abstract}
Oil palm has become well known for its oil palm yields that can be used to produce food, biodiesel and biogas. The rapid expansion of oil palm plantations over large areas has changed the land use and land cover of surroundings. Changes in land covers can be mapped and later used for further analysis. However, obtaining and classifying large coverages require massive amounts of data and computing resources and the skills and time of analysts. The Remote Ecosystem Monitoring Assessment Pipeline (REMAP) provides a cloud computing platform that hosts an open-source stacked Landsat data that allows land cover classification to be implemented using a built-in random forest supervised machine learning algorithm. Classifications were performed with the aid of predictor layers to discriminate the following land covers in Peninsular Malaysia: oil palm, built-up, bare soil, water, forest, other vegetation and paddy. The classification performed on period 1 (1999-2003) and period 2 (2014-2017) data produced an overall accuracy of $80.34 \%$ and $79.53 \%$ respectively. The analysis of the changes in oil palm distributions from period 1 to period 2 indicated an increment of $23.59 \%$. Further analysis revealed that oil palm expansion in Peninsular Malaysia only minimally affected forested area and is mostly resulted from the conversion of less productive crops to oil palm. Results prove the land cover mapping and change detection capabilities of REMAP as a cloud computing platform for large areas. Despite its limitations, REMAP has the potential to achieve fast-paced mapping over large areas and monitor land changes in oil palm distributions.
\end{abstract}

